\title{
EFFICIENT SAMPLING IN EVENT-DRIVEN ALGORITHMS FOR REACTION-DIFFUSION PROCESSES
}

\author{
HOSSEIN BANI-HASHEMIAN ${ }^{1}$, STEFAN HELLANDER $^{1}$, PER LÖTSTEDT $^{1}{ }^{*}$
}

October 27, 2011

\author{
${ }^{1}$ Division of Scientific Computing, Department of Information Technology \\ Uppsala University, P. O. Box 337, SE-75105 Uppsala, Sweden \\ emails: m.h.banihashemian@gmail.com, stefan.hellander@it.uu.se, perl@it.uu.se
}

\begin{abstract}
In event-driven algorithms for simulation of diffusing, colliding, and reacting particles, new positions and events are sampled from the cumulative distribution function (CDF) of a probability distribution. The distribution is sampled frequently and it is important for the efficiency of the algorithm that the sampling is fast. The CDF is known analytically or computed numerically. Analytical formulas are sometimes rather complicated making them difficult to evaluate. The CDF may be stored in a table for interpolation or computed directly when it is needed. Different alternatives are compared for chemically reacting molecules moving by Brownian diffusion in two and three dimensions. The best strategy depends on the dimension of the problem, the length of the time interval, the density of the particles, and the number of different reactions.
\end{abstract}

Keywords: event-driven algorithm, chemical reactions, diffusion, efficient sampling.

AMS subject classification: 65C05, 65C35, 82C80.

PACS subject classification: 02.50.Ey, 02.70.Uu, 83.10.Rs, 87.10.Rt.

\section{Introduction}

It is of interest in many fields of physics, chemistry, biology, and medicine to simulate the dynamical evolution of particles diffusing independently in three dimensional space according to Brownian dynamics and interact with each other

*Financial support has been obtained from the Swedish Research Council. Corresponding author: Per Lötstedt, address as above, telephone +46-18-4712972, fax +46-18-523049. 
when they are adjacent to each other in kinetic Monte Carlo (KMC) algorithms. Each particle is tracked individually and they may coalesce with a certain probability when they collide or are close together and can split into two products. Examples are found e.g. in $[1,5,8,12,13,16,17]$.

The simulation technique is usually categorized as either time-driven or eventdriven [7]. In a time-driven algorithm, the particles in the system are advanced in time by small time steps $\Delta t$, processing events such as collisions and reactions at the end of the time step. The time step is usually longer in an event-driven simulation where $\Delta t$ is the time between two events in the system but there is also more computational work in each step. The situation is somewhat similar in the numerical integration of stiff ordinary differential equations. An explicit method is easy to program but needs small $\Delta t$ for stability and an implicit method is more complicated but allows longer $\Delta t$. A new event or the new position of a particle is found by sampling a probability distribution. This distribution is Gaussian for a particle in free space but for two particles in the neighborhood of each other with a risk of collision or reaction between them, the distribution is more complicated. The cumulative distribution function (CDF) is required for the sampling using the inverse transform sampling algorithm and it is sometimes known analytically. Since the number of time steps is large and there may be many particles, the sampling has to be computationally efficient.

In this paper, we compare different ways of sampling the distribution in an event-driven process for simulation of biochemical reactions and diffusion. The molecules are assumed to be hard and spherical and are partitioned into single molecules and pairs of molecules as in the Green's Function Reaction Dynamics (GFRD) algorithm by van Zon and ten Wolde [19]. The mathematical model for the diffusing and reacting molecules was proposed by Smoluchowski [14]. The probability density function (PDF) for the positions of two molecules in a pair satisfies a parabolic partial differential equation (PDE). The boundary conditions for the PDE are given by Collins and Kimball [6]. If the molecules react, then an associative event has occurred and a new molecule is created. Another event is when a molecule dissociates into two molecules. Single molecules move inside protective spheres where the risk of collision with other molecules is very small.

While random sampling for a single molecule is simple, it is more complicated for a pair of molecules. The center coordinates of a pair are found by sampling a normal distribution and the PDF of the distance between the molecules is determined by the Smoluchowski equation. The differential operator in this PDE is split into two or three parts corresponding to the coordinate directions in [9]. The sampling is considerably simplified in this way at the cost of a numerical splitting error. The CDF in each step may be known analytically or can be computed numerically from an analytically known or numerically determined PDF. If the CDF is expensive to compute every time it is needed, then it can be tabulated and the relative position of the molecules in the pair is computed by interpolation in the table. In principle, these are the alternatives for the 
computational procedure in every event-driven algorithm based on sampling of a CDF.

Different strategies are evaluated for sampling of the radial distance and the angular direction between two molecules in two and three dimensions (2D and $3 \mathrm{D})$. The accuracy in the alternatives is at the same level and the computing time is measured and compared. The algorithms are implemented in MATLAB and executed on a laptop. The difference is significant in some cases. If the CDF is tabulated, then there is an initial cost to compute the table but the sampling using interpolation in a table is much cheaper than computing the CDF several times for each sampling point. Depending on the length of the time interval and the number of molecules, the cost of generating the table may or may not be justified.

The contents of the paper are organized as follows. In the next section, the Smoluchowski equation and the boundary conditions for the PDF are presented. The operator splitting and the different solution methods for the PDE in spherical and cylindrical coordiantes are discussed in Section 3. The efficiency of the sampling techniques is investigated in numerical experiments in Section 4. The best approach for 3D simulation is chosen in Section 5 to verify the correctness of the algorithm. Conclusions are drawn in Section 6. More details and data for a sphercial coordinate system are found in [2].

\section{The Smoluchowski equation}

Consider a reversible association and dissociation reaction

$$
A+B \underset{k_{d}}{\stackrel{k_{a}}{\rightleftharpoons}} C
$$

where $k_{a}$ and $k_{d}$ are the association and dissociation rates, respectively. Depending on the value of $k_{a}, A$ and $B$ may immediately react with each other to form molecule $C$ when their distance is equal to the reaction radius $\sigma$. Dissociation of $C$ into $A$ and $B$ occurs with the rate $k_{d}$. We assume that molecule $A$ is at a fixed position in $3 \mathrm{D}$ at the origin of a spherical coordinate system $(r, \theta, \phi)$ and along the $z$-axis in a cylindrical coordinate system $(r, \phi, z)$. Molecule $B$ is free to move randomly in space by diffusion with a diffusion coefficient $D$, which is defined to be the sum of the diffusion coefficients in free space of $A$ and $B$. In the spherical system, both molecules are sphere-shaped and in the cylindrical system, $A$ is cylindrical and $B$ is spherical.

\subsection{Spherical coordinates}

Let $\mathbf{r}=(r, \theta, \phi)^{\mathbf{T}}$ be the position vector of a point in $3 \mathrm{D}$. The time dependent conditional $\mathrm{PDF}$ of the molecule $B$ to be at position $\mathbf{r}$ at time $t$ starting at 
position $\mathbf{r}_{0}$ at time $t_{0}<t, p_{\mathbf{r}}\left(\mathbf{r}, t \mid \mathbf{r}_{0}, t_{0}\right)$, satisfies the Smoluchowski equation

$$
\partial_{t} p_{\mathbf{r}}=D\left(\frac{\partial^{2} p_{\mathbf{r}}}{\partial r^{2}}+\frac{2}{r} \frac{\partial p_{\mathbf{r}}}{\partial r}+\frac{1}{r^{2} \sin \theta} \frac{\partial}{\partial \theta}\left(\sin \theta \frac{\partial p_{\mathbf{r}}}{\partial \theta}\right)+\frac{1}{r^{2} \sin ^{2} \theta} \frac{\partial^{2} p_{\mathbf{r}}}{\partial \phi^{2}}\right),
$$

with initial condition

$$
p_{r}\left(\mathbf{r}, t_{0} \mid \mathbf{r}_{0}, t_{0}\right)=\delta\left(\mathbf{r}-\mathbf{r}_{0}\right),
$$

and boundary conditions

$$
\begin{aligned}
& \lim _{r \rightarrow \infty} p_{\mathbf{r}}\left(r, \theta, \phi, t \mid \mathbf{r}_{0}, t_{0}\right)=0 \\
& \left.4 \pi \sigma^{2} D \frac{\partial p_{\mathbf{r}}}{\partial r}\right|_{r=\sigma}=\left.k_{a} p_{\mathbf{r}}\left(\mathbf{r}, t \mid \mathbf{r}_{0}, t_{0}\right)\right|_{r=\sigma}-k_{d}\left(1-S\left(t \mid \mathbf{r}_{0}, t_{0}\right)\right) .
\end{aligned}
$$

The probability of the molecules being bound together as $C$ at $t$ is $p_{r}\left(*, t \mid \mathbf{r}_{0}, t_{0}\right)$ and is computed via

$$
p_{\mathbf{r}}\left(*, t \mid \mathbf{r}_{0}, t_{0}\right)=\left.\int_{t_{0}}^{t} \int_{0}^{2 \pi} \int_{0}^{\pi} \sigma^{2} \sin \theta D \frac{\partial p_{\mathbf{r}}\left(\mathbf{r}, \tau \mid \mathbf{r}_{0}, t_{0}\right)}{\partial r}\right|_{r=\sigma} d \theta d \phi d \tau .
$$

The survival probability $S$ or the probability that no reaction occurs between the molecules in $\left[t_{0}, t\right]$ is defined as follows:

$$
\begin{aligned}
S\left(t \mid \mathbf{r}_{0}, t_{0}\right) & =1-p_{r}\left(*, t \mid \mathbf{r}_{0}, t_{0}\right) \\
& =\int_{\sigma}^{\infty} \int_{0}^{2 \pi} \int_{0}^{\pi} \rho^{2} \sin \theta p_{\mathbf{r}}\left(\rho, \theta, \phi, t \mid \mathbf{r}_{0}, t_{0}\right) d \theta d \phi d \rho .
\end{aligned}
$$

In the case of an irreversible reaction, $k_{d}=0$, the boundary condition (2.5) is simplified to

$$
\left.4 \pi \sigma^{2} D \frac{\partial p_{\mathbf{r}}}{\partial r}\right|_{r=\sigma}=\left.k_{a} p_{\mathbf{r}}\left(\mathbf{r}, t \mid \mathbf{r}_{0}, t_{0}\right)\right|_{r=\sigma}
$$

When $k_{a}=0$ in (2.8) we have a homogeneous Neumann boundary condition at $r=\sigma$ and there is no associative reaction and $B$ bounces away from $A$ and as $k_{a} \rightarrow \infty$, we have absorption and $\left.p_{\mathbf{r}}\left(\mathbf{r}, t \mid \mathbf{r}_{0}, t_{0}\right)\right|_{r=\sigma}=0$.

\subsection{Cylindrical coordinates}

Consider an elongated $A$ molecule such as a polymer or a molecular chain along the polar $z$ axis in $3 \mathrm{D}$ in a cylindrical coordinate system. The position of $B$ is at $(r, \phi, z)$ and the Smoluchowski equation for the PDF is

$$
\partial_{t} p_{\mathbf{r}}=D\left(\frac{\partial^{2} p_{\mathbf{r}}}{\partial r^{2}}+\frac{1}{r} \frac{\partial p_{\mathbf{r}}}{\partial r}+\frac{1}{r^{2}} \frac{\partial^{2} p_{\mathbf{r}}}{\partial \phi^{2}}+\frac{\partial^{2} p_{\mathbf{r}}}{\partial z^{2}}\right)
$$

The initial condition is (2.3) and the solution vanishes when $r \rightarrow \infty$ as in (2.4). At $r=\sigma$ we consider only association of $B$ with $A$

$$
\left.2 \pi \sigma D \frac{\partial p_{\mathbf{r}}}{\partial r}\right|_{r=\sigma}=\left.k_{a} p_{\mathbf{r}}\left(\mathbf{r}, t \mid \mathbf{r}_{0}, t_{0}\right)\right|_{r=\sigma}
$$




\section{Position sampling techniques}

The Smoluchowski PDEs (2.2) and (2.9) are solved using operator splitting or fractional steps and the resulting CDFs after each splitting step are sampled consecutively in the coordinate directions in [9]. Very complicated formulas for the analytical solution, see e.g. [4, 19], and high dimensional interpolation in tables are avoided in this way. A time step $\Delta t$ is chosen and a straightforward application of the splitting technique introduces an error of $\mathcal{O}(\Delta t)$ in the PDE solution. A refined splitting due to Strang reduces the error to $\mathcal{O}\left(\Delta t^{2}\right)[15]$.

The differential operator in the right hand sides of (2.2) and (2.9) is decomposed into two or three parts. The solution is determined analytically or numerically and integrated analytically or numerically to obtain the CDF in the first coordinate direction. Then a position $X$ is sampled in that direction using the CDF $F(X)$ and inverse transform sampling. Let $\xi$ be uniformly distributed in $[0,1]$. Then $X$ satisfies $\xi=F(X)$. The procedure is then repeated for the remaining directions.

\subsection{Spherical coordinates}

The splitting steps in spherical coordinates are:

(i) a radial part with derivatives only in the radial direction:

$$
\partial_{t} p_{r}=D\left(\frac{\partial^{2} p_{r}}{\partial r^{2}}+\frac{2}{r} \frac{\partial p_{r}}{\partial r}\right)
$$

with initial condition

$$
p_{r}\left(r, t_{0} \mid r_{0}, t_{0}\right)=\delta\left(r-r_{0}\right),
$$

a vanishing solution when $r \rightarrow \infty$ and the boundary condition

$$
4 \pi \sigma^{2} D \frac{\partial p_{r}}{\partial r}=k_{a} p_{r}\left(\sigma, t \mid r_{0}, t_{0}\right)-k_{d}\left(1-S\left(t \mid r_{0}, t_{0}\right)\right)
$$

at $r=\sigma$ with the survival probability $S$ defined in (2.7). Determine the $\mathrm{CDF}$ and sample for $r$ at $t_{0}+\Delta t$.

(ii) an angular part with derivatives in the polar and azimuthal directions:

$$
\partial_{t} p_{\theta}=\frac{D}{r^{2}}\left(\frac{1}{\sin \theta} \frac{\partial}{\partial \theta}\left(\sin \theta \frac{\partial p_{\theta}}{\partial \theta}\right)+\frac{1}{\sin ^{2} \theta} \frac{\partial^{2} p_{\theta}}{\partial \phi^{2}}\right),
$$

with $\theta \in[0, \pi], \phi \in[0,2 \pi)$ and the initial condition

$$
p_{\theta}\left(\theta, \phi, t_{0} \mid r, 0, \phi_{0}, t_{0}\right)=\delta(\theta) \text {. }
$$

The solution $p_{\theta}$ is independent of $\phi$. Thus, compute the CDF and sample it for the new $\theta$. The new azimuthal angle at $t_{0}+\Delta t$ is obtained by sampling the uniform distribution. 


\subsubsection{Solution and sampling in the radial direction}

\section{Analytical solution}

In [10], an analytical solution to (3.1) - (3.3) is derived. By looking for a solution of the form $p_{r}=f\left(r, t \mid r_{0}\right)+g(r, t)$ where $f$ is the solution for reflective boundary conditions they obtain

$$
\begin{aligned}
& p_{r}\left(r, t \mid r_{0}, t_{0}\right) 4 \pi r r_{0} \sqrt{D}=\frac{1}{\sqrt{4 \pi t}}\left\{\exp \left(-R_{1}^{2}\right)+\exp \left(-R_{2}^{2}\right)\right\} \\
& +B(\alpha, \beta, \gamma, t)+B(\beta, \gamma, \alpha, t)+B(\gamma, \alpha, \beta, t)
\end{aligned}
$$

where

$$
\begin{gathered}
R_{1}=\frac{\left(r-r_{0}\right)^{2}}{4 D t}, R_{2}=\frac{r+r_{0}-2 \sigma}{\sqrt{4 D t}} \\
B(\alpha, \beta, \gamma, t)=\frac{\alpha(\gamma+\alpha)(\alpha+\beta)}{(\gamma-\alpha)(\alpha-\beta)} \exp \left(2 a b+b^{2}\right) \operatorname{erfc}(a+b) .
\end{gathered}
$$

The complex complementary error function is denoted by erfc and $-\alpha,-\beta$ and $-\gamma$ are the roots of the equation

$$
\sigma x^{3}+\sqrt{D}\left(1+\frac{k_{a}}{k_{D}}\right) x^{2}+\sigma k_{d} x+\sqrt{D} k_{d}=0
$$

with $k_{D}=4 \pi \sigma D$. In [11] they show that the CDF, $F_{p_{r}}$, is given by

$$
\begin{aligned}
& F_{p_{r}}\left(r, t \mid r_{0}, t_{0}\right)+\left(1-S\left(t \mid r_{0}, t_{0}\right)\right)=-\frac{\sqrt{D t}}{r_{0} \sqrt{\pi}}\left\{\exp \left(R_{1}^{2}\right)-\exp \left(-R_{2}^{2}\right)\right\} \\
& +\frac{1}{2}\left\{\operatorname{erf}\left(R_{1}\right)+\operatorname{erf}\left(R_{2}\right)\right\}+E(\alpha) B(\alpha, \beta, \gamma, t)+E(\beta) B(\beta, \gamma, \alpha, t) \\
& +E(\gamma) B(\gamma, \alpha, \beta, t)
\end{aligned}
$$

where erf is the error function, $E(x)=\frac{1}{r_{0} x}\left(r-\frac{\sqrt{D}}{x}\right)$, and $S\left(t \mid r_{0}, t_{0}\right)$ is the survival probability in (2.7).

Given two molecules at a distance $r_{0}$ at time $t_{0}$ we can now sample the new distance $r$ at time $t$ with inverse transform sampling by inverting the CDF numerically. In Figure 3.1 we see how the PDF and CDF change with different values of $r_{0}$. If the uniformly distributed $\xi>\max _{r} F_{p_{r}}$, then a reaction between $A$ and $B$ has occurred and they are replaced by $C$.

\section{Numerical solution}

To solve the problem (3.1) - (3.3) numerically, we use the Crank-Nicolson finite difference scheme to obtain $p_{i}^{n}$ at the $i^{\text {th }}$ grid point and time $t^{n}$ because of its 

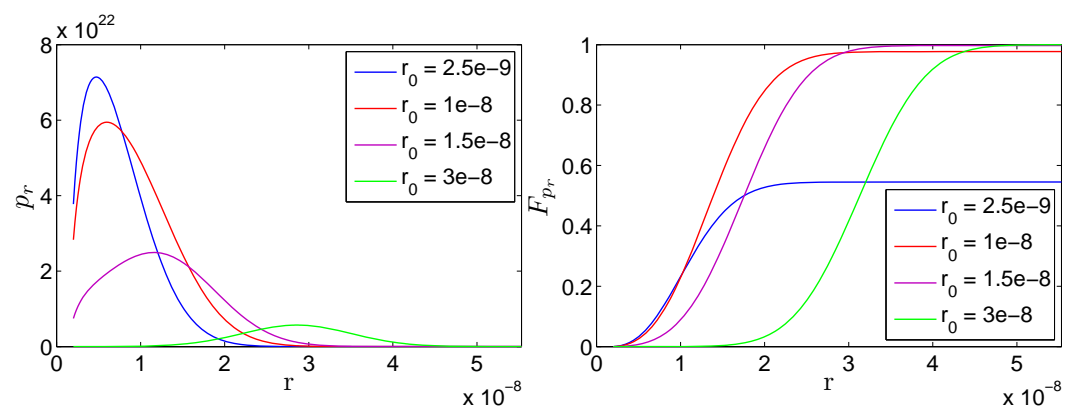

Figure 3.1: The PDF and CDF in the radial direction for different values of $r_{0}$. The model parameters are $k_{a}=10^{-19}, k_{d}=2.9894, D=2 \cdot 10^{-12}, \sigma=2 \cdot 10^{-9}, \Delta t=10^{-5}$.

stability and second order accuracy in time and space. The grid is non-uniform in space with step size $h_{j}$, always containing the initial position $r_{0}$, and uniform in time with time step $\Delta \tau<\Delta t$. The initial condition (3.2) is approximated by $p_{j}^{0}=0, j \neq i$, and $p_{i}^{0}=1 / h_{i}$ where $i$ is the index of $r_{0}$ in the spatial grid.

The boundary condition at infinity is replaced by the same condition at a suitably large finite distance $r_{\max }$. The distance a particle can travel can be approximated by that of a freely diffusing particle. Thus, a reasonable choice is $r_{\max }=c \sqrt{2 D \Delta t}$ for some constant $c$. We have found that $c=4$ is sufficiently large.

The boundary condition (3.3) is approximated at $t=t^{n}$ by

$$
4 \pi \sigma^{2} D \frac{p_{2}^{n}-p_{1}^{n}}{h_{2}}=k_{a} p_{1}^{n}-k_{d}\left(1-S\left(t^{n}\right)\right), \quad 1 \leqslant n \leqslant N
$$

where the survival probability $S\left(t^{n}\right)$ is integrated with the trapezoidal rule using $p_{i}^{n-1}$. Finally the CDF is approximated, again using the trapezoidal rule.

The most critical requirement for implementing the numerical scheme is that $r_{0}$ must be one of the discretization points in the $r$ direction, due to its presence in the initial condition. In order to have such a grid in space, the subinterval $\left[\sigma, r_{0}\right]$ is first discretized into $M_{\text {left }}=10$ points. Then using the same step size we discretize the subinterval $\left[r_{0}, r_{\max }\right]$. To obtain a sufficiently accurate solution we may then have to refine the grid to the left of $r_{0}$.

When the initial position $r_{0}$ is close to the reaction radius $\sigma$, the numerical solution exhibits a decaying oscillatory behaviour around $r_{0}$ unless $\Delta \tau$ is sufficiently small. Because of this the numerical method is not recommended when the molecules are located initially in or too close to the bound state. One way of resolving the issue with the oscillatory behaviour is to use an adaptive grid algorithm, but then a major effort is necessary for the implementation. 


\section{Interpolation in a precomputed lookup table}

Using either of the methods above we precompute a $3 \mathrm{D}$ lookup table in $r_{0}, \Delta t$, and $r$ for the CDF with fixed model parameters $k_{a}, k_{d}, \sigma$ and $D$ to be used for the approximate sampling of the distribution. This method involves two stages: creating the lookup table and performing interpolation between the data points in the table.

When creating the table we first choose $n_{r_{0}}$ points in the direction of $r_{0}$ and $n_{t}$ points in the direction of $\Delta t$. Then the CDF is computed at discrete $r$-values for every $n_{r_{0}} \cdot n_{t}$ possible combination of the $r_{0}$ and $\Delta t$ values using either the numerical scheme, by computing the analytical solution given by (3.10) or by computing the analytical PDF given by (3.6) and then the CDF by numerical integration.

If the table is generated by one of the analytical formulas, we can use a fixed number of points in $r$-space, but when creating the table with the numerical method the spatial grid will depend on $r_{0}$. Then we need to keep an additional table with information about the points in the $r$-direction and their CDF values.

Now, for given $r_{0}$ and $\Delta t$, a new $r$ is determined by first retrieving the four points in the table surrounding $r_{0}$ and $\Delta t$ with their corresponding CDFs. Then a $\xi$ uniformly distributed in $[0,1]$ is sampled, and finally we compute $r_{\xi}$ by performing trilinear interpolation in the table. The interpolation region, i.e. the table cell containing $\xi$ from the CDF of interest, can have a very irregular shape. This is due to the difference in the curvature of the graphs of the four neighbouring CDFs, a part of which forms four sides of the cell. In Figure 3.2, the panel to the left represents such a cell in one of the worst cases.

A feasible interpolation technique for computing $r_{\xi}$ is via two bilinear interpolations between the vertices of the faces ${ }_{1} p_{1} p^{\prime} p_{1}^{\prime} p_{1}$ and ${ }_{2} p_{2} p^{\prime} p_{2}^{\prime} p_{2}$ of the cell, see Figure 3.2, followed by one linear interpolation resulting in a trilinear interpolation, but in the general case the vertices do not have the same radial coordinate $r$. One way to handle this problem is to interpolate inside a regularly cube-shaped region circumscribing the original cell (as the right panel in Figure 3.2 illustrates). Then the new vertices ${ }_{1} P{ }_{1} P^{\prime}, P_{1}^{\prime}$, and $P_{1}$ have the same $r$ coordinate equal to $r^{(1)}:=\min \left\{r_{1}, r_{p_{1}}, r_{1} p^{\prime}, r_{p_{1}^{\prime}}\right\}$, and the same situation holds for ${ }_{2} P{ }_{2} P^{\prime}$, $P_{2}^{\prime}$, and $P_{2}$ where $r=r^{(2)}:=\max \left\{r_{2}, r_{p_{2}}, r_{2} p^{\prime}, r_{p_{2}^{\prime}}\right\}$. The CDF values at these new points are also needed, which can be calculated by linear interpolation.

Now that all the conditions for performing the bilinear interpolations are satisfied, we find two points $Q$ and $R$ normally located on opposite sides of the target point, in the sense that they all belong to the same CDF and $\xi$ is between the values of the cumulative distribution function at $Q$ and $R$, or $F_{Q} \leq \xi \leq F_{R}$. Considering $Q$ and $R$ as interpolation points, $r_{\xi}$ is obtained by linear interpolation. If $\xi$ is not between $F_{Q}$ and $F_{R}, r_{\xi}$ is computed via linear extrapolation.

Here we create two lookup tables using $200 \times 200$ pairs of sampled $r_{0}$ and $\Delta t$ values. The computed analytical $\mathrm{CDF}$ values are stored in one table and 

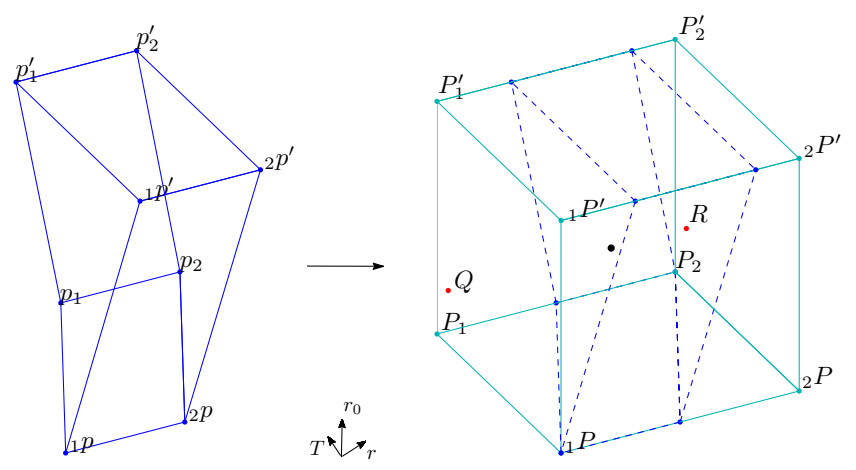

Figure 3.2: A cell from the 3D lookup table whose entries are the CDF values obtained from numerical method. This cell contains a data point with value $\xi=0.9595$. The result from interpolation is shown for $r_{0}=8.8962 \cdot 10^{-8}$ and $\Delta t=1.2558 \cdot 10^{-4}$, and the model parameters are fixed as $k_{a}=10^{-19}, k_{d}=2.9894, D=2 \cdot 10^{-12}, \sigma=2 \cdot 10^{-9}$.

the other one contains the numerical values. For fixed $r_{0}$ and $\Delta t$, we used the same spatial discretization in $r$ for both the analytical and the numerical method. The number of time steps $\Delta \tau$ to reach $\Delta t$ in the numerical scheme is changed according to a piecewise constant function of $r_{0}$ to accelerate the computations.

\section{Interpolation without lookup table}

When computing the CDF analytically or numerically for given $r_{0}$ and $\Delta t$ without storing it in a table we still need to invert it numerically to perform inverse transform sampling. To this end the CDF is computed on a grid $r_{1}<r_{2}<$ $\ldots<r_{N}$ and then for a given $\xi$ we find $i$ such that $F^{-1}\left(r_{i}\right) \leq \xi \leq F^{-1}\left(r_{i+1}\right)$ by performing a linear search. Finally, to find $r_{\xi}$ such that $F^{-1}\left(r_{\xi}\right) \approx \xi$ is found by inverse linear interpolation.

\subsubsection{Solution and sampling in the angular direction}

Applying finite difference schemes to solve the problem (3.4), (3.5), involves difficulties due to the singularity in the equation at $\theta=0$. This is the reason why we will only compare computing the analytical solution directly with linear interpolation in a precomputed look-up table.

\section{Analytical solution}

The analytical solution of the angular part of the Smoluchowski equation (3.4), (3.5), is given in the form of an infinite series, see e.g. [18, 21], 


$$
p_{\theta}\left(\theta, t_{0}+\Delta t \mid r, 0, \phi_{0}, t_{0}\right)=\sum_{l=0}^{\infty} \frac{2 l+1}{4 \pi r^{2}} \exp \left(-l(l+1) \frac{D \Delta t}{r^{2}}\right) P_{l}(\cos \theta),
$$

where $P_{l}$ denotes the $l^{\text {th }}$-degree Legendre polynomial. Note that for a fixed radial distance $r$, the conditional PDF (3.12) is a function of the polar angle $\theta$ and time $t$ and independent of the azimuthal angle $\phi$. The convergence of the series in (3.12) can be verified analytically using standard techniques and the rate of convergence is mainly influenced by the value of the parameter $\hat{t}=D \Delta t / r^{2}$. The PDF for different values of $\hat{t}$ is plotted in Figure 3.3. As we can see, $p_{\theta}$ tends to a uniform distribution as $\hat{t}$ grows.
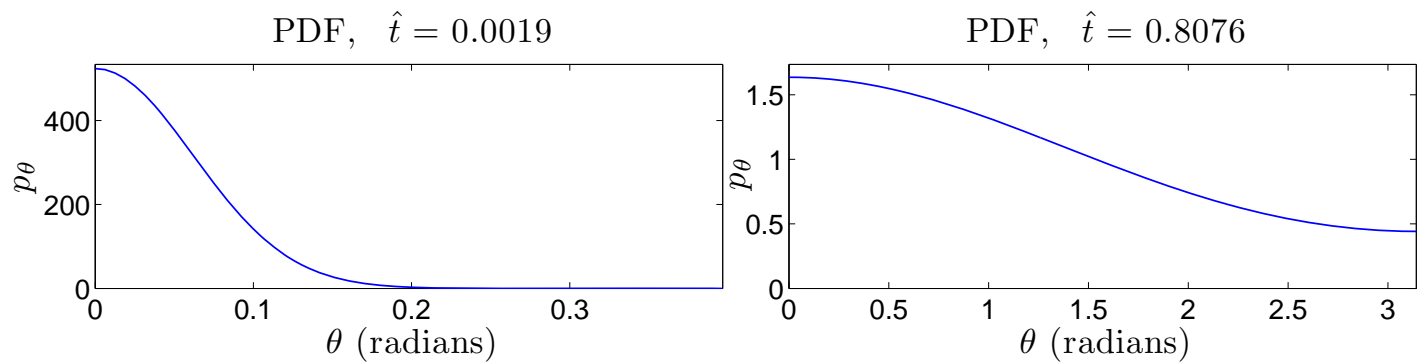

Figure 3.3: The PDF in the polar direction for two different values of $\hat{t}$.

For practical computations, the infinite series (3.12) has to be truncated to a finite summation. A possible choice of the level of truncation is the first positive integer $l_{\max }$ for which the absolute value of the remainder term

$$
\mathcal{R}=\sum_{l=l_{\max }+1}^{\infty}(2 l+1) \exp (-l(l+1) \hat{t}) P_{l}(\cos \theta)
$$

is small. Since $\left|P_{l}(x)\right| \leq 1$ for any $x \in[-1,1]$ and every positive integer $l$, we have

$$
|\mathcal{R}| \leq \sum_{l=l_{\max }+1}^{\infty}(2 l+1) \exp (-l(l+1) \hat{t}) .
$$

Approximating the sum in (3.14) by an infinite integral and requiring that the integral is less than a small number $\epsilon$, gives the following criterion

$$
-\left(l_{\max }+1\right)\left(l_{\max }+2\right) \hat{t} \leq \ln \hat{t} \epsilon,
$$

for truncating (3.12) at a proper level. 
The criterion (3.14) is expressed in terms of $\hat{t}$ and for a fixed value of $\hat{t}$, we can estimate the PDF $p_{\theta}$ at a number of not necessarily equidistant grid points for $\theta \in[0, \pi]$. However, for $\hat{t}$ small $p_{\theta}$ decays quickly with growing $\theta$ and unnecessary computations are avoided by introducing another stopping criterion given by

$$
\left|a_{l_{\max }}\right| \leq \epsilon^{\prime}\left|s_{l_{\max }}\right|
$$

Here, $\epsilon^{\prime}$ is small and positive, $a_{l_{\max }}$ is the $l_{\max }$-th term of the series (3.12), and $s_{l_{\max }}$ denotes the sum of the series after truncation at the index $l_{\max }$. When this criterion is met the PDF has stopped changing within some given relative tolerance $\epsilon^{\prime}$.

In Figure 3.4, the total number of terms needed to reach the stopping criterion (3.15) with $\epsilon=10^{-5}$ is plotted against $\theta$ and the natural logarithm of $\hat{t}$. The series converges slowly for small values of $\hat{t}$ (in the worst case we need 3049 terms) but when $\hat{t}$ is large the stopping criterion is fulfilled after only a few terms. However, when $\hat{t}$ is small the criterion in (3.16) is reached quickly and the number of points where $p_{\theta}$ is computed in the $\theta$-direction is small. We have chosen $\epsilon^{\prime}=0.2$.

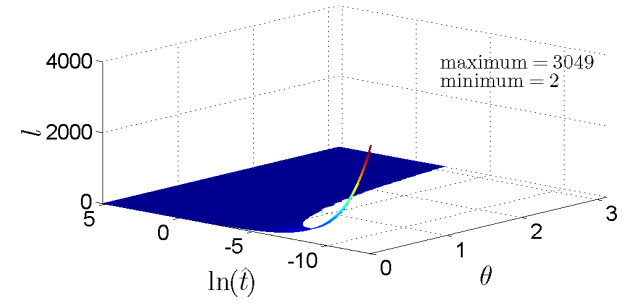

Figure 3.4: The number of terms $l=l_{\max }+1$ of the infinite series (3.12) sufficient to reach convergence with $\epsilon=10^{-5}$ are displayed as a function of $\theta$ and $\ln \hat{t}$. When $\ln \hat{t}$ is small the stopping criterion (3.16) is reached quickly, seen as the blank areas in the figure.

Having the PDF $p_{\theta}$ obtained as described above, the corresponding CDF is evaluated numerically using the trapezoidal rule and a polar position of a molecule can be sampled via inverse transform sampling.

\section{Interpolation in a precomputed lookup table}

The second approach employed to sample polar positions is to perform linear interpolation between precomputed CDF values tabulated in a 2D lookup table. This method consists of two stages: creating the lookup table and linear interpolation between the entries of the table.

The procedure of creating the CDF lookup table is as follows. Assume that we have a set of $n_{t}$ preselected but not necessarily equally spaced values $\hat{t}_{i}$ in 
a suitable range, sorted in increasing order. For every member of this set and on a fixed grid consisting of $n_{\theta}$ points in the polar direction, we compute the corresponding CDF via the method described in the previous section. Depending on the value of $\hat{t}$ and the stopping criteria, the $n_{t}$ vectors of the precomputed CDF values may have different lengths of at most $n_{\theta}$ forcing us to introduce a data structure similar to what was proposed in Section 3.1.1 for the radial direction.

After creating the table, for any arbitrary value of $r$ and $\Delta t$ for which $\hat{t}_{1} \leq$ $D \Delta t / r^{2} \leq \hat{t}_{n_{t}}$ we find the four surrounding data points forming a parallelogramshaped region. A new polar position is then sampled from the CDF by bilinear interpolation.

\subsection{Cylindrical coordinates}

The dimensional splitting in cylindrical coordinates is:

(i) a radial part, with derivatives only in the radial direction:

$$
\partial_{t} p_{r}=D\left(\frac{\partial^{2} p_{r}}{\partial r^{2}}+\frac{1}{r} \frac{\partial p_{r}}{\partial r}\right)
$$

with the same initial condition as in (3.2), a vanishing solution when $r \rightarrow \infty$, and a boundary condition at $r=\sigma$

$$
2 \pi \sigma D \frac{\partial p_{r}}{\partial r}=k_{a} p_{r}\left(\sigma, t \mid r_{0}, t_{0}\right)
$$

Determine the CDF from $p_{r}$ and sample for $r$ at $t_{0}+\Delta t$.

(ii) in the axial and azimuthal directions, the new positions are obtained by sampling normal distributions in the coordinates $z$ and $r \phi$ with the angle chosen modulo $2 \pi$.

The solution of (3.17) is also the PDF of the relative distance between two molecules moving on a flat $2 \mathrm{D}$ membrane and $\phi$ is the angular direction.

\subsubsection{Solution and sampling in the radial direction}

\section{Analytical solution}

For the radial part of the Smoluchowski equation (3.17) and (3.18), the following exact analytical solution is available in [4, p. 370]:

$$
\begin{aligned}
p_{r}\left(r, t_{0}+\Delta t \mid r_{0}, t_{0}\right) & =\frac{1}{2 \pi} \int_{0}^{\infty} \exp \left(-D u^{2} \Delta t\right) \mathcal{C}\left(u, r, r_{0}, k, k_{a}\right) u d u \\
\mathcal{C}\left(u, r, r_{0}, k, k_{a}\right) & =C\left(u, r, k, k_{a}\right) C\left(u, r_{0}, k, k_{a}\right)
\end{aligned}
$$


where $k=2 \pi l \sigma D$ and the function $C$ is defined as a combination of Bessel functions of order $n$ of the first and second kind $J_{n}$ and $Y_{n}, n \in\{0,1\}$,

$$
\begin{aligned}
C\left(u, r, k, k_{a}\right) & =\frac{J_{0}(u r) \alpha(u)-Y_{0}(u r) \beta(u)}{\left(\alpha(u)^{2}+\beta(u)^{2}\right)^{1 / 2}}, \\
\alpha(u) & =k u Y_{1}(\sigma u)+k_{a} Y_{0}(\sigma u), \quad \beta(u)=k u J_{1}(\sigma u)+k_{a} J_{0}(\sigma u) .
\end{aligned}
$$

The CDF is given by

$$
F_{p_{r}}\left(r, t_{0}+\Delta t \mid r_{0}, t_{0}\right)=\int_{\sigma}^{r} 2 \pi \rho p_{r}\left(\rho, t_{0}+\Delta t \mid r_{0}, t_{0}\right) d \rho
$$

and can be determined either by computing the exact PDF (3.19) and then the CDF by numerical integration or by using the analytical formula for the CDF directly for an approximation.

\section{Computing the PDF}

The integrand in (3.19) inherits the complex oscillatory behaviour of $C$, see Figure 3.5, but the term $u e^{-D u^{2} \Delta t}$ acts as a damping factor decaying rapidly after its maximum at $u=\sqrt{1 / 2 D \Delta t}$. The domains of $r$ and $u$ are truncated in the calculations to the finite intervals $\left[\sigma, r_{\max }\right]$ and $\left[0, u_{\max }\right]$, respectively,

$$
F_{p_{r}}\left(r, t_{0}+\Delta t \mid r_{0}, t_{0}\right) \approx \int_{\sigma}^{r_{\max }} 2 \pi \rho \int_{0}^{u_{\max }} u e^{-D u^{2} \Delta t} \mathcal{C}\left(u, r, r_{0}, k, k_{a}\right) d u d \rho
$$

where $r_{\max }=r_{0}+4 \sqrt{2 D \Delta t}$ and $u_{\max }=\sqrt{\frac{1}{D \Delta t} \ln \left(\frac{1}{2 \epsilon K D \Delta t}\right)}$ is chosen to satisfy the condition $K \int_{u_{\max }}^{\infty} u e^{-D u^{2} \Delta t} d u \leq \epsilon$ for a small positive $\epsilon$ assuming that $\left|\mathcal{C}\left(u, r, r_{0}, k, k_{a}\right)\right| \leq K$. Due to the complicated form of $\mathcal{C}$, it is difficult to find an exact upper bound $K$. Nevertheless for our parameter values and based on observations from a set of graphs, $K=1$ appears to be a quite satisfactory choice.

The outer integral in (3.24) is computed by the trapezoidal rule and for the inner integral, an adaptive quadrature scheme based on the Gauss-Kronrod rule is employed as implemented in MATLAB's built-in routine quadgk. Using algebraic transformations, quadgk can also handle the singularity of the integrand at $u=0$.

Depending on the values of $r_{0}$ and $\Delta t$, the truncations and approximations explained above may result in oscillations in the computed PDF solution when $r<r_{0}$. There, the PDF remains at a constant level just before it starts growing to its peak. To lessen the effect of artificial oscillations, we replace the lower limit of the outer integral by $r_{\min }=\min \left\{\sigma, r_{0}-4 \sqrt{2 D \Delta t}\right\}$ and assume that if $r_{\min }>\sigma$ then $p_{r}\left(r, t \mid r_{0}, t_{0}\right)=p_{r}\left(r_{\min }, t \mid r_{0}, t_{0}\right)=0$ for $r \in\left[\sigma, r_{\min }\right]$. 

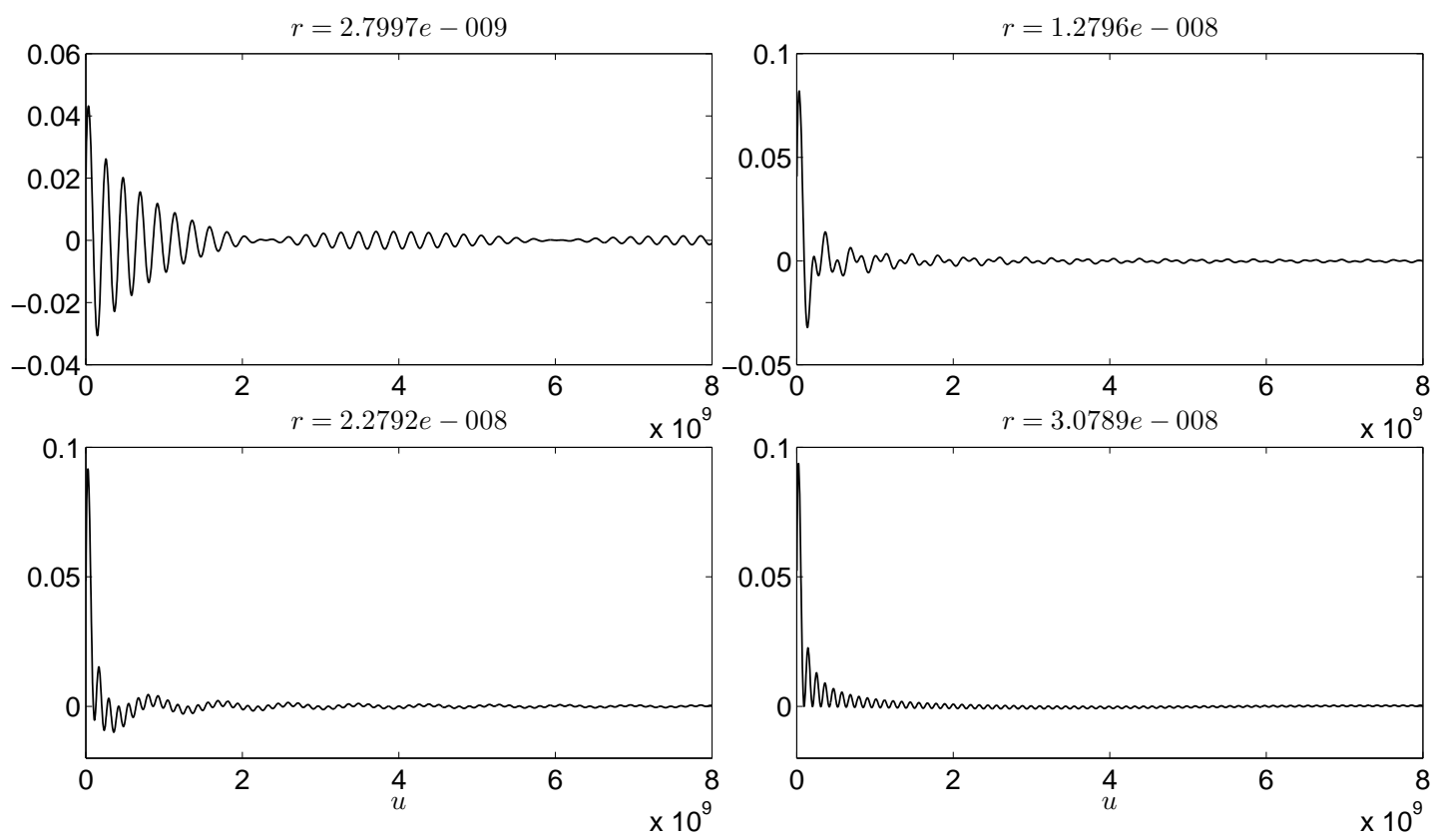

Figure 3.5: The oscillatory behaviour of the function $\mathcal{C}\left(u, r, r_{0}, k, k_{a}\right)$ for four different values of $r$ with $r_{0}=3 \cdot 10^{-8}$ and $\Delta t=10^{-7}$.

\section{Computing the semi-analytical CDF}

After truncating the $r$ domain at a finite distance $r_{\max }=r_{0}+4 \sqrt{2 D \Delta t}$ and a change of the order of integration and using that $\int_{a}^{b} r A_{0}(r) d r=b A_{1}(b)-a A_{1}(a)$ with $A=J$ or $Y$ we can rewrite (3.23) as

$$
F_{p_{r}}\left(r, t \mid r_{0}, t_{0}\right)=\int_{0}^{\infty} e^{-D u^{2} \Delta t} R(u) d u
$$

where

$$
\begin{aligned}
R(u) & =\frac{C\left(u, r_{0}, k, k_{a}\right) \gamma(u)}{\left(\alpha(u)^{2}+\beta(u)^{2}\right)^{1 / 2}}, \\
\gamma(u) & =\alpha(u)\left(r_{\max } J_{1}\left(r_{\max } u\right)-\sigma J_{1}(\sigma u)\right)-\beta(u)\left(r_{\max } Y_{1}\left(r_{\max } u\right)-\sigma Y_{1}(\sigma u)\right) .
\end{aligned}
$$

Since no closed expression of (3.25) is known to us, we approximate the integral numerically. First, the upper limit of the integral is replaced by a finite value $u_{\max }$. The integrand exhibits oscillations, whose amplitudes tend to zero as $u$ tends to infinity. Since the oscillations are difficult to predict, we assume that $|R(u)| \leq K^{\prime}$. Choose $u_{\max }$ such that

$$
\operatorname{erf}\left(u_{\max } \sqrt{D \Delta t}\right) \geq 1-\frac{2 \epsilon \sqrt{D \Delta t}}{K^{\prime} \sqrt{\pi}}
$$


and approximate the error function by

$$
\operatorname{erf}(x) \approx\left(1-\exp \left(-x^{2} \frac{\frac{4}{\pi}+0.14 x^{2}}{1+0.14 x^{2}}\right)\right)^{\frac{1}{2}}
$$

as in [20]. Then with $u_{\max }$

$$
K^{\prime} \int_{u_{\max }}^{\infty} e^{-D u^{2} \Delta t} d u \leq \epsilon
$$

where $u_{\max }$ is the real positive root of a biquadratic equation of the form $c_{1} u^{4}+$

$c_{2} u^{2}+c_{3}=0$, where $c_{1}=0.14 D \Delta t, c_{3}=\frac{1}{D \Delta t} \ln \left(1-\left(1-\frac{2 \epsilon \sqrt{D \Delta t}}{K^{\prime} \sqrt{\pi}}\right)^{2}\right), c_{2}=$ $\frac{4}{\pi}+c_{1} c_{3}$ and $K^{\prime}=10^{-3}$.

Some artificial oscillations may appear in the calculated CDF for small $r_{0}$ but they are avoided by defining a lower integration limit $r_{\min }=\min \left\{\sigma, r_{0}-4 \sqrt{2 D \Delta t}\right\}$ as in the previous section.

The look-up table is precomputed either by first computing the PDF in (3.19) and then the CDF using the trapezoidal rule or using the analytic expression in (3.25) for the CDF. The new radial position is found by interpolation in the table as in Section 3.1.1.

\section{Comparison of sampling methods}

In this section, we compare the sampling methods in Section 3 with respect to accuracy and efficiency. To check the accuracy, we assume that the analytical methods yield the most accurate results, although some approximation errors are involved also there. Another assumption is that the quality of the coding of the algorithms is at the same level. It is possible that a comparison is altered by an improved implementation but in many cases the outcome is clear and the opposite conclusion is unlikely. We performed all the experiments in MATLAB v7.10 (R2010a) on a laptop PC with an Intel Core 2 Duo T7200 processor $(2.00 \mathrm{GHz}$, 4MB L2 Cache) and 2GB of RAM running Windows 7 (64 bit).

\subsection{Spherical coordinates}

The methods for sampling the distributions in spherical coordinates are evaluated using 92225 distinct pairs of values for the initial radial position $r_{0}$ and time step $\Delta t$. The values are collected from a simulation of one trajectory of a system using the method in [9]. In this way, we get a fair distribution of samples when comparing the overall accuracy and efficiency. For convenience we denote the set of the pairs of values by PoVsph. The model parameters in (2.2) and (2.5) are $k_{a}=10^{-19}, k_{d}=2.9894, D=2 \cdot 10^{-12}$, and $\sigma=2 \cdot 10^{-9}$. 


\subsubsection{The radial direction}

The different methods in Section 3.1.1 to solve (3.1), (3.2), and (3.3) for the PDF and integration of it for the CDF are compared in measurements of their accuracy and efficiency.

\section{Accuracy comparison}

We begin by comparing the sampling methods with respect to accuracy. The comparison is made by applying all the methods over the whole set of values PoVsph. Very few members of the set whose $r_{0}$ values are very close to $\sigma$ are excluded because then we are unable to use the numerical method. A uniformly distributed $\xi$ is chosen. Then the relative error in per cent in computing $r_{\xi}$ by inverse transform sampling is collected for all pairs of $r_{0}$ and $\Delta t$ of the set. The error in a method is assumed to be the difference between $r_{\xi}$ obtained with the method and $r_{\xi}$ from the analytical method. To investigate the error data, the data are split into 21 groups according to the $r_{0}$ values and averaged over all $\Delta t$ values in the group. The conclusions from the comparisons are the same if the data are instead averaged over the $r_{0}$ values.

The results are displayed using box-and-whisker plots (or box-plots for short) as demonstrated in Figure 4.1. In these plots, each box encloses the middle $50 \%$ of the data, i.e. the bottom and top edges of the box are the $25^{\text {th }}$ and $75^{\text {th }}$ quartiles, $q_{25}$ and $q_{75}$, of the data, respectively. The whiskers or dashed T-bars display the highest and the lowest data points not considered to be outliers. With the interquartile range $I_{q r}=q_{75}-q_{25}$, the outliers are defined as values $x$ such that $x<q_{25}-1.5 I_{q r}$ or $x>q_{75}+1.5 I_{q r}$. The line across the box represents the median and the cross, diamond, or asterisk in the box indicates the mean value which is computed excluding the outlier values.

From the box-plots in Figure 4.1, we find that the numerical method with the chosen mesh size is more accurate than the lookup table (lut) interpolation methods based on numerical or analytical data. Except for the leftmost $r_{0}$-interval, the lut-interpolation method performs quite well and the difference between the two versions of the lut-interpolation method is small. The two tables are stored on the same grid. Thus, the difference is due to the error from solving the equations numerically. For small values of $r_{0}$ or $\Delta t$, a large variation is noticed in the relative error due to a few data points in the table. The errors are positively or right skewed with the mean and the median being only a few per cent.

When two molecules stay close to each other for a while, then $r_{0}$ and $\Delta t$ are small and errors may accumulate over several small time steps. This issue can be conclusively resolved by using high-resolution lookup tables containing more CDFs for small initial particle positions and small time intervals. Other sources of error that influence the accuracy of the lut-interpolation method are: the error in computing the entries of a lookup table and the error introduced 

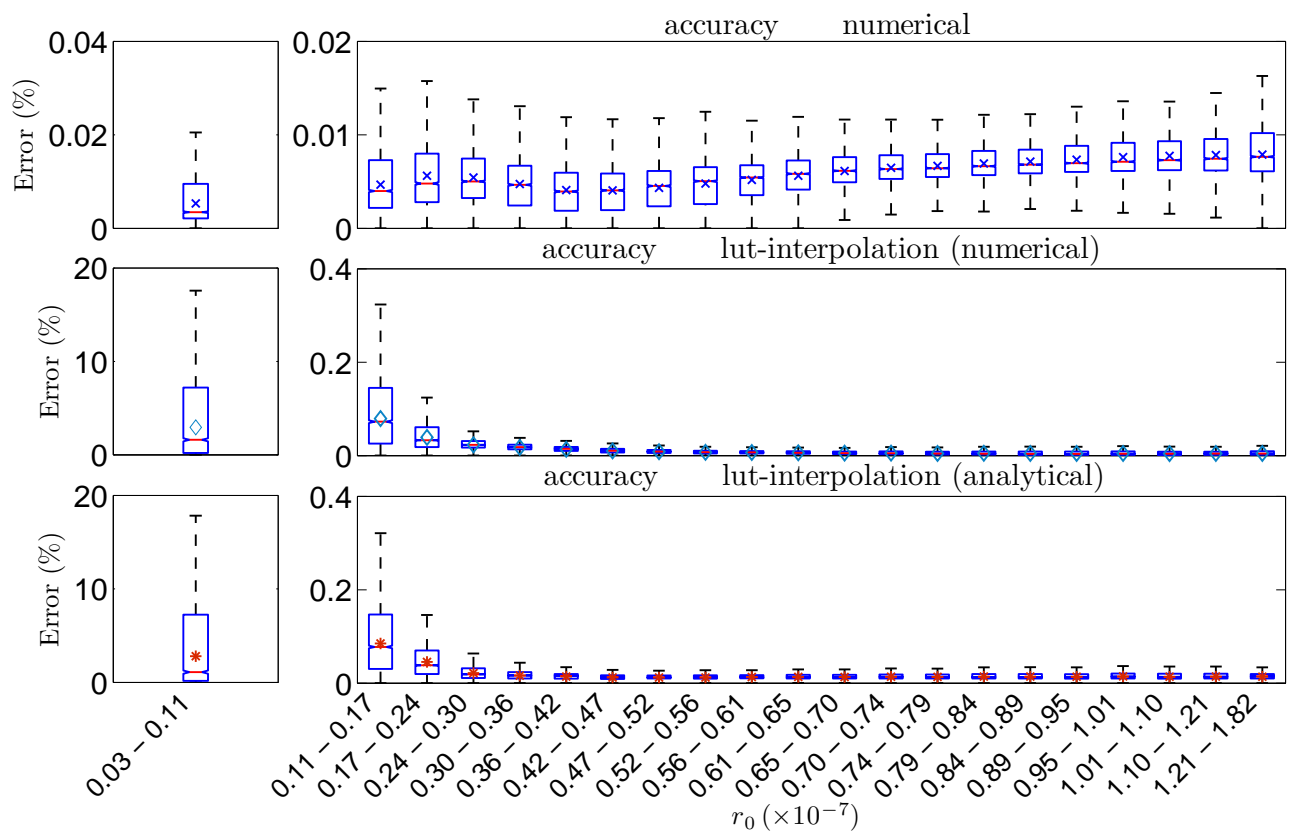

Figure 4.1: Box-plots of percent error in computing $r_{\xi}$ in spherical coordinates using different sampling methods versus $r_{0}$. The experiment is performed for 91735 distinct pairs of values for $r_{0}$ and $\Delta t$ with $\xi=0.5469$.

by transforming the original region of interpolation in the lookup table into a regularly cube-shaped region (see Figure 3.2).

\section{Efficiency comparison}

The required CPU time to compute every single solution that we used in our accuracy experiment in the previous section is measured in Figure 4.2. The data are presented as in Figure 4.1. The accuracy of the methods is about the same in Figure 4.1 but the numerical method, with an average run time of $0.172 \mathrm{~s}$, is noticeably less efficient than the other methods. The conclusion from the plots is that among the sampling methods the analytical method with numerical integration, which yields the sampled position on average within $2.24 \mathrm{~ms}$, is the fastest one. This is approximately 77 times faster than the numerical method and 1.85 times faster than the lut-interpolation method with $4.16 \mathrm{~ms}$ average CPU time per run.

The speed of the numerical method is highly dependent on the grid size in the spatial domain and the total number of time steps used to obtain the numerical PDF. When the initial position $r_{0}$ is close to $\sigma$, then we have to use more grid points in the radial direction to have an accurate solution requiring more time steps to avoid oscillatory solutions. This explains why the numerical method is 


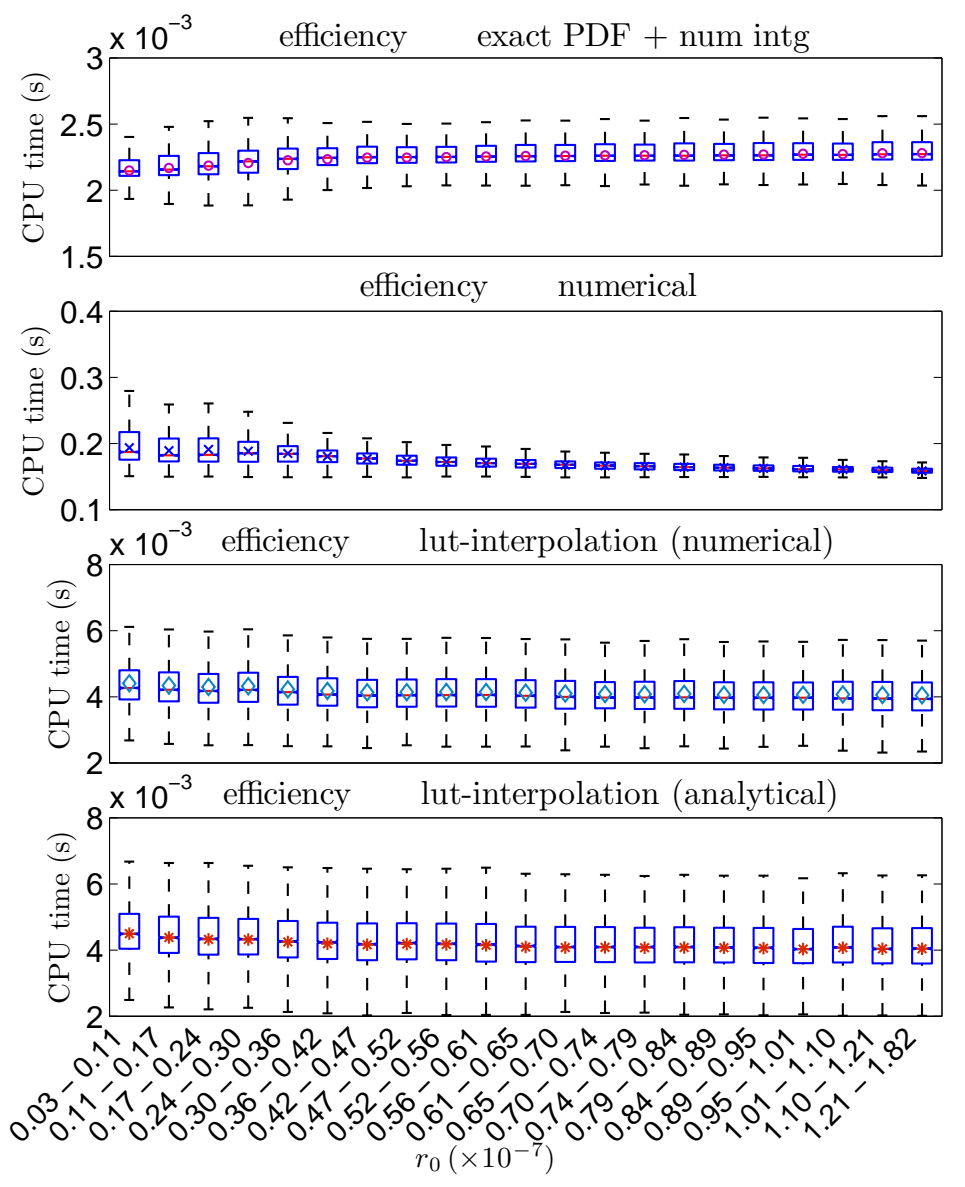

Figure 4.2: Box-plots of the CPU time required to compute $r_{\xi}$ in spherical coordinates using different sampling methods versus $r_{0}$. Here $\xi=0.5469$ and the experiment is performed for 91735 distinct pairs of values of $r_{0}$ and $\Delta t$. For every pair, the computations are repeated up to 10 times.

marginally slower in the very first sub-ranges of values for $r_{0}$. Adaptively refined grids and time steps would resolve this issue in an efficient manner.

The CDF in the table is computed in two different ways: by numerical integration of the PDF or by the analytical formula (3.10). The PDF is obtained by numerical solution of the Smoluchowski equation (3.1) or by the analytical formula (3.6). There are 200 pairs of values of $r_{0}$ in $\left[3 \cdot 10^{-9}, 2.06 \cdot 10^{-7}\right]$ and of $\Delta t$ in $\left[1 \cdot 10^{-8}, 7.6 \cdot 10^{-4}\right]$ in the table. For each $\left(r_{0}, \Delta t\right)$ entry, there are several $r$ values and corresponding CDF values. The size of the table is $52.7 \mathrm{MB}$. The conclusion from Table 4.1 is that the most efficient way to create the table is to use the analytical expression for the PDF and then integrate it numerically. 


\begin{tabular}{|l|c|}
\hline Method & CPU time (min) \\
\hline PDF by numerical solution & 15.34 \\
\hline Analytical PDF & 1.45 \\
\hline Numerical integration of PDF for CDF & 0.03 \\
\hline Analytical CDF & 1.90 \\
\hline
\end{tabular}

Table 4.1: The CPU time for different parts of the generation of a table.

\subsubsection{The angular direction}

Two approaches are proposed in Section 3.1.2 to draw random polar positions determined by the solution of the angular part of the Smoluchowski equation in (3.4) and (3.5): a method with an analytically computed PDF and then numerically integrated and interpolation between the CDF values precomputed and stored in a lookup table.

\section{Accuracy comparison}

The accuracy of the lut-interpolation method for $\theta$ is estimated by comparisons with the solutions obtained by the analytical method. Here we fix $\xi$ and calculate 92225 distinct values for the parameter $\hat{t}=D \Delta t / r^{2}$ using all the pairs of values of $\Delta t$ and $r$ in the set PoVsph. Then for every value of $\hat{t}$, we compute $\theta_{\xi}$ using both sampling techniques and the relative error in the result obtained from the lut-interpolation method.

The error data are partitioned into 21 sub-ranges based on $\ln \hat{t}$ in Figure 4.3. In the box-plot, it is obvious that the accuracy of the lut-interpolation method is very high with an average relative error of $0.008 \%$. An example of the dependency of the accuracy on the resolution of the precomputed lookup table is observed for small $\hat{t}$-values. For those values, the slope of the $p_{\theta}$ at $\theta=0$ is large and to obtain a smooth, high-resolution CDF, a finer grid in the polar direction close to $\theta=0$ is needed.

\section{Efficiency comparison}

The efficiency of the analytical method is compared with the lut-interpolation method by measuring the CPU time to compute the solutions in the above accuracy analysis in Figure 4.4. The accuracy of the two methods is comparable in Figure 4.4. The methods are also compared to the sampling method in [3]. There a random walk on a sphere $\mathcal{S}^{2}$ in $\mathbb{R}^{3}$ is generated directly by summation of the series for random walk on the sphere $\mathcal{S}^{3}$ embedded in $\mathbb{R}^{4}$ and projection to the sphere $\mathcal{S}^{2}$. The method in [3] determines a $\theta$ sample directly with a good accuracy for small $\hat{t}$ while only a few terms are needed in the series in (3.12) for large $\hat{t}$ and there its accuracy is good. The average runtime of the lut-interpolation method is $0.58 \mathrm{~ms}$ and for the projection method $0.35 \mathrm{~ms}$. It is clear that the 


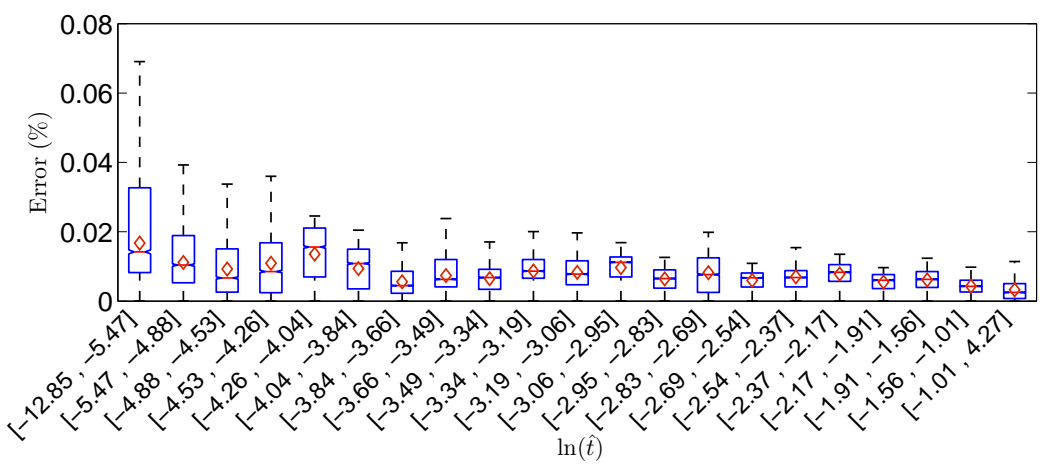

Figure 4.3: Box-plot of percentage error in computing $\theta_{\xi}$ using lut-interpolation methods versus $\ln \hat{t}$. Here $\xi=0.8147$ and the experiment is performed for 92225 distinct values of $\hat{t}$.

lut-interpolation method is much more efficient than the analytical method but that the algorithm in [3] is the fastest when it works for small $\hat{t}$.

In Section 3.1.2, we observed that for small values of the parameter $\hat{t}$, evaluating $p_{\theta}$ via its analytical formula (3.12) can be computationally very challenging since many terms of the infinite series have to be summed to reach convergence. The problem becomes less severe for larger values of $\hat{t}$ explaining the gradual decrease in the mean CPU time for the analytical method as $\hat{t}$ increases in Figure 4.4 .

The CDF in the table for interpolation in Figure 4.4 is computed at $300 \hat{t}$ values where $\ln \hat{t}$ is between -12.85 and 5.23. The CDF is evaluated for at most $500 \theta$ values for each $\hat{t}$. The total number of CDF data values in the table is 76862 requiring $483 \mathrm{~KB}$ of memory. The elapsed CPU time for its calculation is $16.7 \mathrm{~s}$.

\subsection{Cylindrical coordinates}

The sampling methods in Section 3.2.1 for cylindrical coordinates are evaluated at 10000 randomly generated distinct pairs of values for the initial radial position $r_{0}$ and time step $\Delta t$ in the set PoVcyl. The parameters in the model in (2.9) and (2.10) are $k_{a}=10^{-11}, D=10^{-12}$, and $\sigma=2 \cdot 10^{-9}$.

\subsubsection{The radial direction}

The analytical method to determine the CDF based on (3.25) and the numerical integration of the PDF in (3.19) in Section 3.2.1 are compared to the lutinterpolation method with precomputed tables of the CDF. 


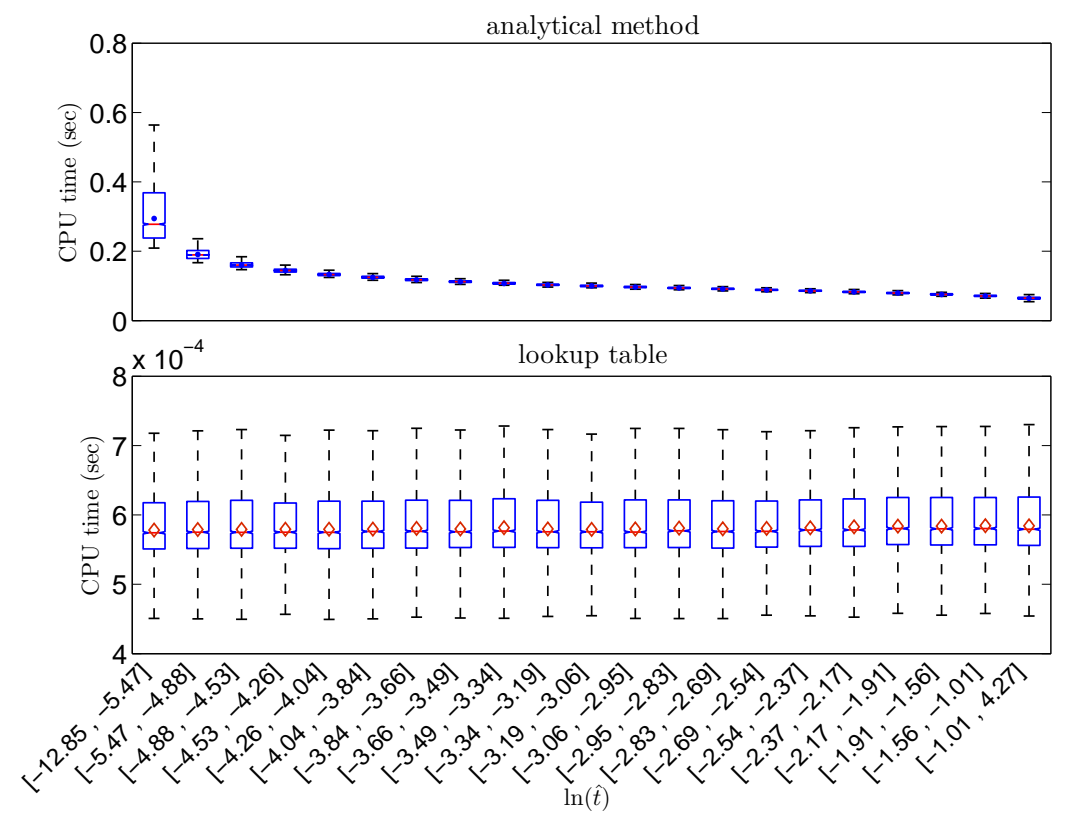

Figure 4.4: Box-plots of the CPU time required to compute $\theta_{\xi}$ using the analytical method and the lut-interpolation method versus $\ln (\hat{t})$. Here $\xi=0.8147$ and the experiment is performed for 92225 distinct values of $\hat{t}$. For every pair computations are repeated up to 10 times.

\section{Accuracy comparison}

The relative errors in Figure 4.5 are determined by comparing the semi-analytical method to the other methods. The data in PoVcyl are grouped according to the value of $r_{0}$ and averaged over all $\Delta t$ values. The mean errors in per cent are very low in most cases. For the smallest $r_{0}$-values, the accuracy in the lut-interpolation is improved by refining the table there similarly to what was necessary for the spherical coordinates.

\section{Efficiency comparison}

Using the $r_{0}$ and $\Delta t$ data in PoVcyl, the efficiency of the sampling methods are compared in Figure 4.6. The average CPU time for the interpolation alternatives is $3.8 \mathrm{~ms}$ and $4.7 \mathrm{~ms}$. The method based on numerical solution of the PDE uses 80 $\mathrm{ms}$ and the semi-analytical method is the worst with $868 \mathrm{~ms}$. The performance of the semi-analytical method deteriorates for large values of $r_{0}$. The reason is that for these values the integrand in (3.25) oscillates with high frequencies making an accurate integration more difficult.

The interpolation table is computed with three methods using the semianalytical CDF, the analytical PDF and numerical integration, and the numerical PDF integrated numerically. The number of CDF data values are between $1.0 \cdot 10^{7}$ 


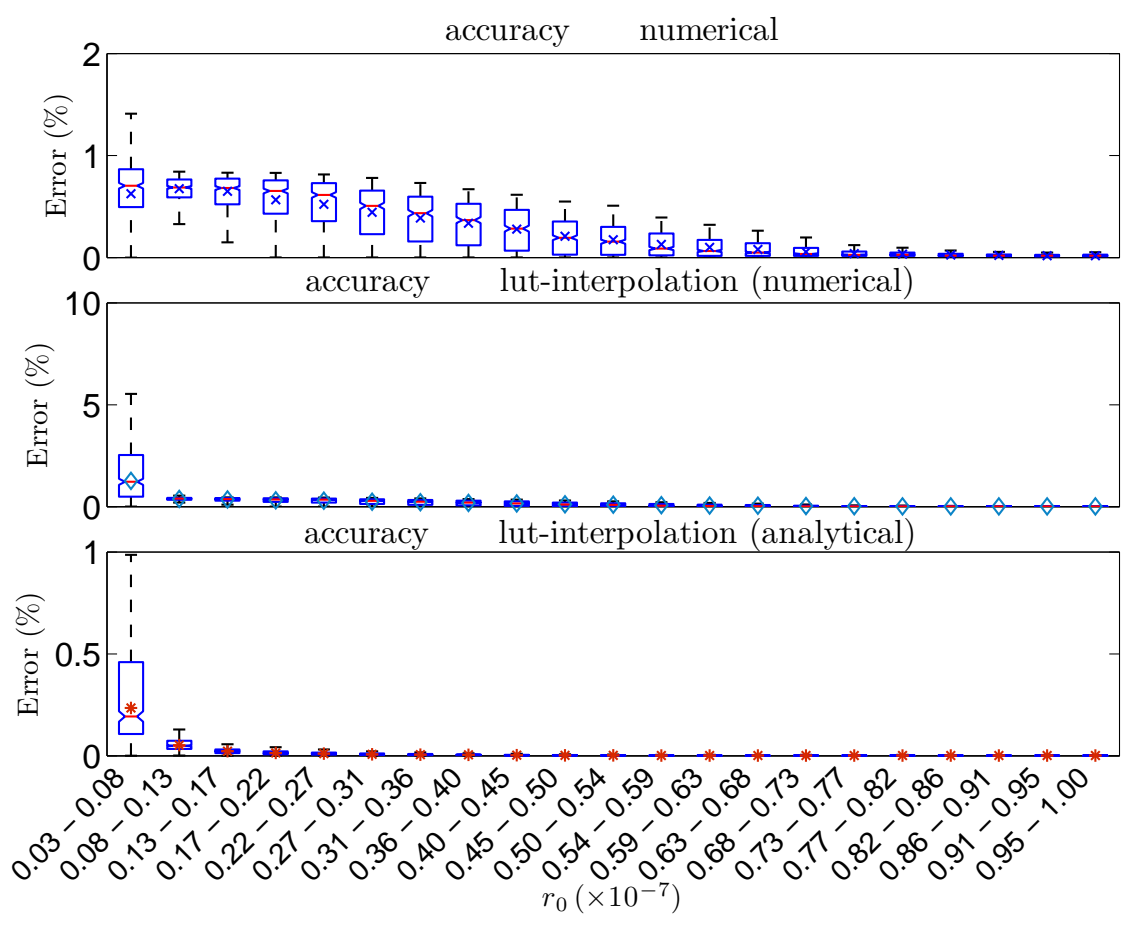

Figure 4.5: Box-plots of per cent error in computing $r_{\xi}$ in cylindrical coordinates using different sampling methods versus $r_{0}$. Here $\xi=0.4505$ and the experiment is performed for 10000 distinct pairs of values for $r_{0}$ and $T$.

and $1.1 \cdot 10^{7}$ requiring between 71 and $73 \mathrm{MB}$ of storage. The difference in memory requirements is small but full numerical integration of the CDF is more than 15 time faster than using analytical formulas in Table 4.2 .

\begin{tabular}{|l|c|}
\hline Method & CPU time (min) \\
\hline Numerical PDF and numerical integration & 28.7 \\
\hline Analytical PDF and numerical integration & 431 \\
\hline Semi-analytical CDF & 455.5 \\
\hline
\end{tabular}

Table 4.2: The CPU time for different ways of generating the table.

\subsection{Sampling for the next reaction event}

Sometimes we want to first sample the time to the next event and then the new positions. For a pair of molecules, the time $t$ to the next reaction satisfies $S\left(t \mid r_{0}\right)=\xi$ for a uniformly distributed $\xi \in[0,1]$ with the survival probability $S$ defined in (2.7). Note that $S\left(t \rightarrow \infty \mid r_{0}\right)<1$ and there is a possibility that a reaction will never occur. In the spherical case $S$ is known analytically [10] and 


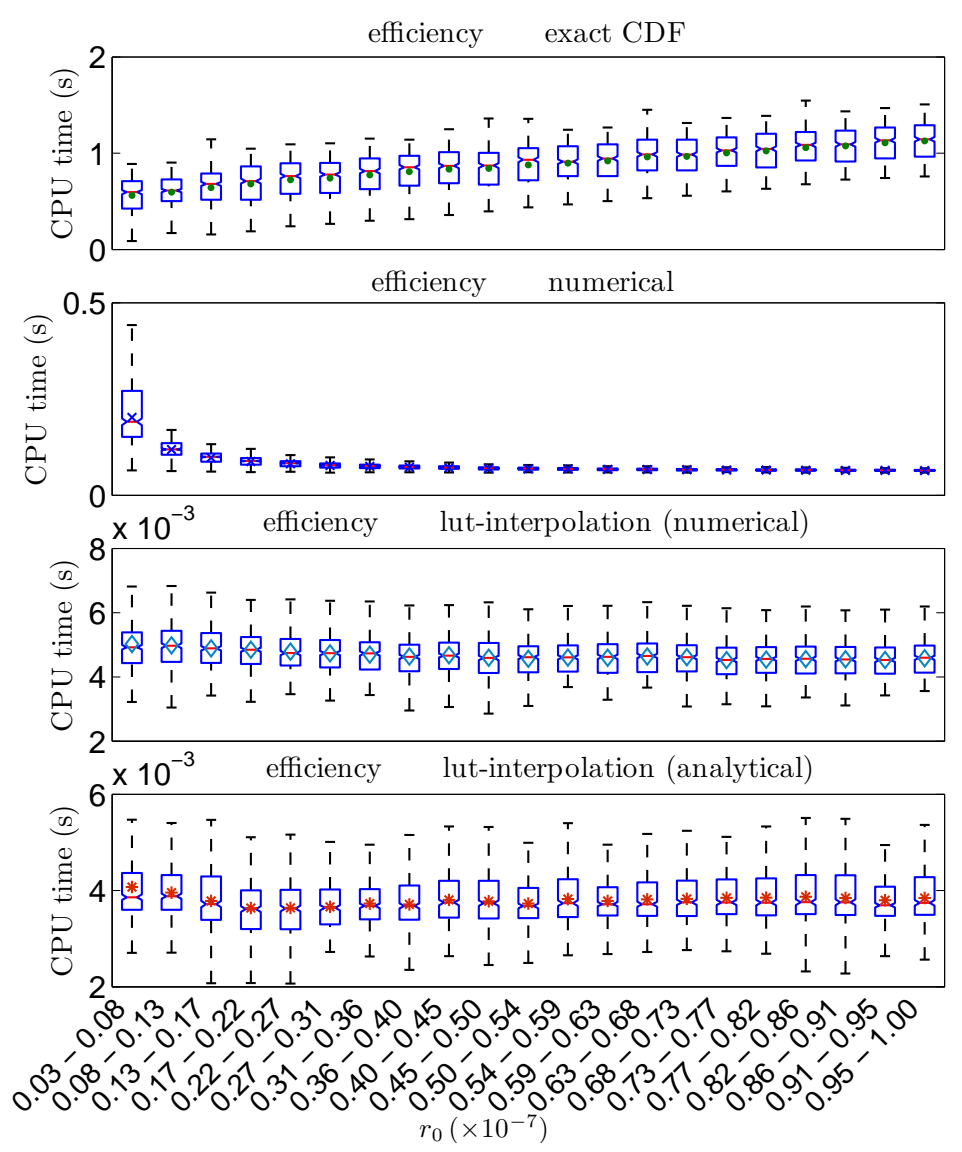

Figure 4.6: Box-plots of the CPU time required to compute $r_{\xi}$ in cylindrical coordinates using different sampling methods versus $r_{0}$. Here $\xi=0.4505$ and the experiment is performed for 10000 distinct pairs of values of $r_{0}$ and $\Delta t$. For every pair computations are repeated up to 10 times.

can be inverted numerically in the same manner as the analytical expression for the CDF. The cost of inverting $S$ is of the same order as inverting the CDF. When computing the PDF numerically, we can compute $S$ after each time step $\Delta \tau$ and determine if the molecules have reacted or not. If two molecules have reacted at a certain time, we can stop the computations and will save some time compared to solving the equation until the final time $\Delta t$. However, the finite difference approach will still very likely be outperformed by the analytical approach. For the analytical approach in the cylindrical case, we can compute $S$ by numerically integrating the expression for the PDF (3.19). This will be a fairly expensive way of inverting $S$, and the conclusion that the numerical approach is more efficient will still hold. 


\subsection{Estimates of total elapsed time}

Suppose that the number of time steps is $N$ in a simulation, that there are $m_{i}$ pairs of particles in step $i$, that the CPU time for sampling for each pair in every step is $t_{\text {sampl }}$, and that the elapsed time between the events is $\Delta t_{i}$. Ignoring single particles for which sampling is inexpensive, the total CPU time for sampling $T_{\text {sampl }}$ and the final time $T$ are then

$$
T_{\mathrm{sampl}}=\sum_{i=1}^{N} m_{i} t_{\mathrm{sampl}}=N \bar{m} t_{\mathrm{sampl}}, \quad T=\sum_{i=1}^{N} \Delta t_{i}=N \overline{\Delta t},
$$

where $\bar{m}$ and $\overline{\Delta t}$ are the average number of pairs in every step and the average time step. Suppose that the CPU time to create one table is $T_{\text {tab }}$ and that there are $R$ reactions. Hence, the total CPU time for generating the tables and simulating the system to $T$ is

$$
R T_{\mathrm{tab}}+t_{\mathrm{sampl}} \bar{m} T / \overline{\Delta t}
$$

Let $\rho$ be the density of particles and $v$ the volume around a single particle or a

pair. The radius of $v$ is proportional to $\sqrt{2 D \overline{\Delta t}}$. Hence, $1 / \rho \sim v \sim \overline{\Delta t}^{3 / 2}$ and we have $\bar{m} \sim \rho$. The conclusion is that $\bar{m} T / \overline{\Delta t} \sim \rho^{5 / 3} T$. If $T$ is large then the cost of computing the table is discounted but if the density of the particles is low then the first term in (4.1) may dominate. As an example, take the polar direction in Section 4.1.2. There $T_{\mathrm{tab}}=16.7 \mathrm{~s}, t_{\mathrm{sampl}}=6 \cdot 10^{-4} \mathrm{~s}$ and the break even point when the analytical method without a table is as efficient is when $\bar{m} T / \overline{\Delta t} \approx 90$, i.e. if $\bar{m}=1$ then 90 time steps suffice. For larger values of $\bar{m} T / \overline{\Delta t}$ the table will win.

\section{Simulation of a pair of molecules}

Based on the accuracy and efficiency analysis in the previous section, the best technique is chosen to generate particle positions for simulation of reactiondiffusion processes in the spherical and the cylindrical coordinate systems. The algorithm from [9] can be outlined as follows:

1. Given $r_{0}$ and $\Delta t$, sample for the new radial position $r$.

2. Given $r$ and $\Delta t$, sample for the new polar angle $\theta$ or the new azimuthal angle $\phi$ in the spherical and cylindrical cases, respectively.

3. Given $r$ and $\Delta t$, sample for the azimuthal angle $\phi$ or the axial position $z$ in the spherical and cylindrical cases, respectively. 

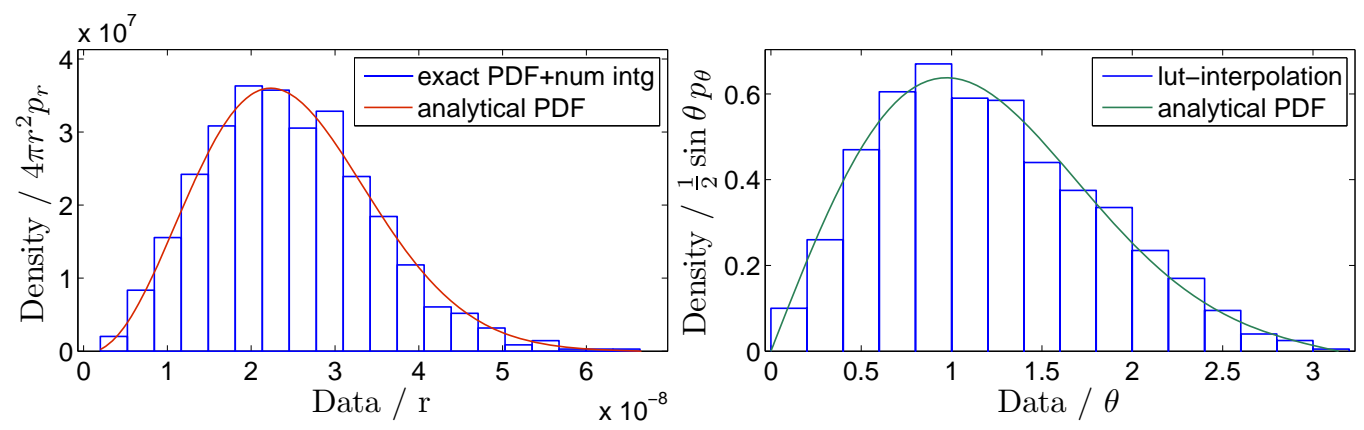

Figure 5.1: The probability densities $4 \pi r^{2} p_{r}$ in the radial direction with $\Delta t=5$. $10^{-5}, r_{0}=10^{-8}$ and $T=5 \cdot 10^{-3}$ (left) and $\frac{1}{2} \sin \theta p_{\theta}$ in the polar direction with $\Delta t=7 \cdot 10^{-4}, r_{0}=5 \cdot 10^{-8}$ and $T=7 \cdot 10^{-2}$ (right) obtained by sampling are compared to the analytical density at $T$.

\subsection{Spherical coordinates}

The optimal method according to Sections 4.1.1 and 4.1.2 in the spherical case is sampling radial positions by numerical integration of the analytical PDF and polar positions using interpolation from precomputed tabulated CDF data. The species $A$ and $B$ react with each other and form $C$ as in (2.1) with $A$ fixed at the origin with a diffusion coefficient $D=2 \cdot 10^{-12}$ and reaction radius $\sigma=2 \cdot 10^{-9}$. We consider two cases: a reversible reaction with $k_{a}=10^{-19}, k_{d}=2.9894$ and free diffusion with $k_{a}=k_{d}=0$.

The new positions of 1000 particles are sampled in the radial and the polar directions and collected in a histogram in Figure 5.1 for the reversible reaction. The weighted $p_{r}$ and $p_{\theta}$ are compared to the analytical solutions with good agreement.

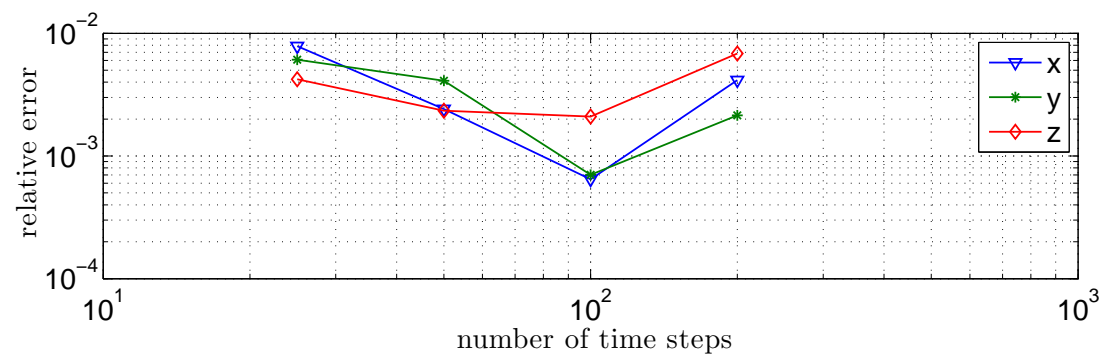

Figure 5.2: The relative difference between the standard deviation of the simulated position and the analytical Gaussian standard deviation for the position of freely diffusing molecules at $T=5 \cdot 10^{-5}$ with $D=2 \cdot 10^{-12}$ using different $\Delta t$.

When no reaction takes place, the new positions in Cartesian coordinates are determined using the above operator splitting algorithm starting at a point away from the origin. One molecule is simulated in a time interval and $2 \cdot 10^{5}$ 
trajectories are generated for each fixed splitting time step $\Delta t$. In the comparison of the computed and analytical standard deviations in Figure 5.2, there is no clear tendency when $\Delta t$ is varied and other errors such as the statistical variation appear to be much larger than the variation due to $\Delta t$. The error is already quite low but to reduce the statistical error further by a factor 10 we would need $2 \cdot 10^{7}$ realizations of the process.

\subsection{Cylindrical coordinates}
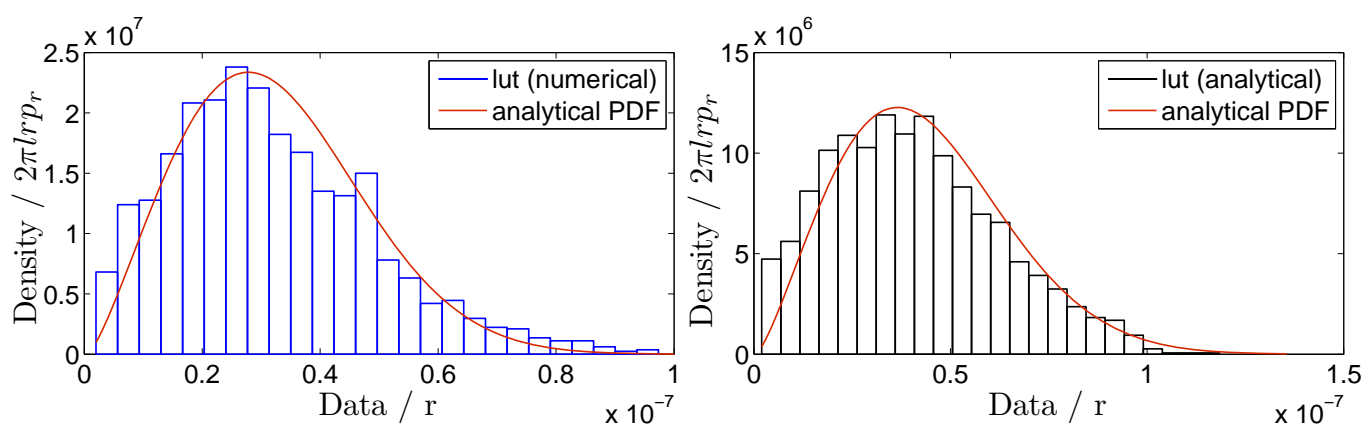

Figure 5.3: The probability densities $2 \pi r \ell p_{r}$ with $\ell=1$ in the radial direction with $\Delta t=2 \cdot 10^{-4}, r_{0}=2 \cdot 10^{-8}$ and $T=1.4 \cdot 10^{-2}$ computed numerically (left) and analytically with $\Delta t=5 \cdot 10^{-4}, r_{0}=9 \cdot 10^{-9}$ and $T=10^{-2}$ (right) are compared to the analytical solution at $T$.

The stationary $A$ molecule is a polymer along the $z$ axis in a cylindrical coordinate system in this example and the $B$ molecule is moving in $3 \mathrm{D}$ space and can react with the polymer by association. The parameters are $k_{a}=10^{-11}, D=$ $10^{-12}$, and $\sigma=2 \cdot 10^{-9}$. The distribution of the radial position is found at the final time $T$ using tabulated data in Figure 5.3 using the statistics from 2000 particles. The accuracy is good for large $r_{0}$, but for $r_{0}$ close to $\sigma$ the accuracy is worse. To obtain good accuracy in this case we may need a very high resolution in the table.

\section{Summary and conclusions}

Event-driven algorithms such as the GFRD algorithm [19] for simulation of biochemical reaction-diffusion processes need fast evaluations of CDFs in the sampling for the next events or the next positions of the particles in the system. The relative positions of two particles in a pair are governed by a PDF satisfying the Smoluchowski equation. We have compared different methods for the sampling when it is split into a radial part and an angular part in a spherical and a cylin- 
drical coordinate system as in [9]. Although we investigate a particular model, the conclusions are of general interest for event-driven algorithms.

The Smoluchowski equation in the radial direction in a spherical coordinate system is solved by an analytical method and the Crank-Nicolson finite difference scheme. The sampling is performed using the analytical CDF of the distribution, the CDF calculated by numerical integration of the analytical PDF, and linear interpolation between the entries of a 3D lookup table with analytically or numerically precomputed CDFs for a set of model parameter values. The lowest CPU time to determine a random radial position is obtained by numerical integration of the PDF. Presumably, the results obtained from this method have the highest accuracy in spite of the fact that the accuracy is influenced by errors introduced by truncating infinite sums and numerical approximations in the integration. Almost as efficient are the methods based on interpolation in a table. A different implementation of the methods might change the recommendation in this case. The explanation why the tables are not the best alternative is that the formulas to compute the PDF and the CDF are relatively simple.

In the polar direction in a spherical coordinate system, the CDF is evaluated partly analytically and partly numerically and there are two approaches to sample from the CDF: using the computed CDF directly and tabulating pre-evaluated CDFs in a 2D lookup table first and then performing linear interpolation between the data points in the table. Using the lookup table is here orders of magnitude faster than the partially analytical method. It is unlikely that this difference is due to the implementation of the methods. The accuracy of the interpolation in the table is very good, but it depends on the resolution in the table. The CDF is here more complicated to compute than in the previous case and tables are the preferred choice. The generation of the table is inexpensive in this case.

The radial direction in a cylindrical coordinate system is sampled with a semi-analytically defined CDF, one computed numerically, and the CDF stored in $3 \mathrm{D}$ tables for inverse interpolation. The tables provide the new $r$ value much faster than the other two methods. It is improbable that this conclusion will be changed by different implementations of the algorithms. The explanation to this result compared to the radial direction in the spherical system is that in the cylinder case the analytical CDF is rather complicated to evaluate with a highly oscillatory integrand while the closed formula in the spherical case has only a few terms. Computing the table entries numerically is here the cheapest method.

In general, using lookup tables is recommended but it depends on the application and the available analytical formulas, the density of the particles, and the amount of time that the simulation will run, as the method has an initial cost in time for creating the tables and a cost in memory space for data storage. One table is needed for each reaction in the biochemical system. Since tables are finite data sets, limitations are imposed on the longest distance that two molecules can move apart and the longest time step that can be taken during the simulation. Furthermore, there is a shortest distance between the initial $r_{0}$ to the reaction 
radius $\sigma$ that a table can resolve with sufficient accuracy. Refinements of the tables are necessary for certain combinations of parameter values. The cost of such refinements are higher the higher the dimension of the table is. This is another argument for splitting the spatial sampling since the dimensions of the tables are usually lower if the sampling in space is made in a sequence of steps.

\section{References}

[1] S. S. Andrews, N. J. Addy, R. Brent, and A. P. Arkin. Detailed simulations of cell biology with Smoldyn 2.1. PLoS Comput. Biol., 6(3):e1000705, 2010.

[2] M. H. Bani-Hashemian. Accurate and Efficient Solution of the Smoluchowski Equation. Master's thesis, Department of Information Technology, Uppsala University, Uppsala, Sweden, 2011. Available online at: http://urn.kb.se/ resolve?urn=urn:nbn: se:uu:diva-156440 .

[3] T. Carlsson, T. Ekholm, and C. Elvingson. Algorithm for generating a Brownian motion on a sphere. J. Phys. A: Math. Theor., 43:505001, 2010.

[4] H. S. Carslaw and J. C. Jaeger. Conduction of Heat in Solids. Oxford University Press, Oxford, second edition, 1959.

[5] J. S. Coggan, T. M. Bartol, E. Esquenazi, J. R. Stiles, S. Lamont, M. E. Martone, D. K. Berg, M. H. Ellisman, and T. J. Sejnowski. Evidence for ectopic neurotransmission at a neuronal synapse. Science, 309:446-451, 2005.

[6] F. C. Collins and G. E. Kimball. Diffusion-controlled reaction rates. J. Colloid. Sci., 4:425-437, 1949.

[7] A. Donev. Asynchronous event-driven particle algorithms. Simulation, 85(4):229-242, 2009.

[8] A. Donev, V. V. Bulatov, T. Oppelstrup, G. H. Gilmer, B. Sadigh, and M. H. Kalos. A first-passage kinetic Monte Carlo algorithm for complex diffusion-reaction systems. J. Comput. Phys., 229:3214-3236, 2010.

[9] S. Hellander and P. Lötstedt. Flexible single molecule simulation of reactiondiffusion processes. J. Comput. Phys., 230(10):3948-3965, May 2011.

[10] H. Kim and K. J. Shin. Exact solution of the reversible diffusioninfluenced reaction for an isolated pair in three dimensions. Phys. Rev. Lett., 82(7):1578-1581, 1999.

[11] H. Kim, K. J. Shin, and M. Yang. Dynamic correlation effect in reversible diffusion-influenced reactions: Brownian dynamics simulation in three dimensions. J. Chem. Phys., 111(3):1068-1075, 1999. 
[12] S. J Plimpton and A. Slepoy. Microbial cell modeling via reacting diffusive particles. J. Physics: Conf. Series, 16(1):305, 2005.

[13] A. Scala, Th. Voigtmann, and C. De Michele. Event-driven Brownian dynamics for hard spheres. J. Chem. Phys., 126(13):134109, 2007.

[14] M. Smoluchowski. Versuch einer mathematischen Theorie der Koagulationskinetik kolloider Lösungen. Z. Phys. Chem., 92:129-168, 1917.

[15] G. Strang. On the construction and comparison of difference schemes. SIAM J. Numer. Anal., 5:506-517, 1968.

[16] K. Takahashi, S. Tănase-Nicola, and P. R. ten Wolde. Spatio-temporal correlations can dratically change the response of a MAPK pathway. Proc. Natl. Acad. Sci. USA., 107(6):2473-2478, 2010.

[17] J. Dalla Torre, J.-L. Bocquet, N. V. Doan, E. Adam, and A. Barbu. JERK, an event-based Kinetic Monte Carlo model to predict microstructure evolution of materials under irradiation. Phil. Mag., 85(4):549-558, 2005.

[18] V. Tulovsky and L. Papiez. Formula for the fundamental solution of the heat equation on the sphere. Appl. Math. Lett., 14:881-884, 2001.

[19] J. S. van Zon and P. R. ten Wolde. Green's-Function Reaction Dynamics: A particle-based approach for simulating biochemical networks in time and space. J. Chem. Phys., 123(23):234910, 2005.

[20] S. Winitzki. Uniform approximations for transcendental functions. In V. Kumar, M. Gavrilova, C. Tan, and P. L'Ecuyer, editors, Computational Science and Its Applications - ICCSA 2003, volume 2667 of Lecture Notes in Computer Science, pages 962-962. Springer, 2003.

[21] K. Yosida. Brownian motion on the surface of the 3-sphere. Ann. Math. Statistics, 20:292-296, 1949. 